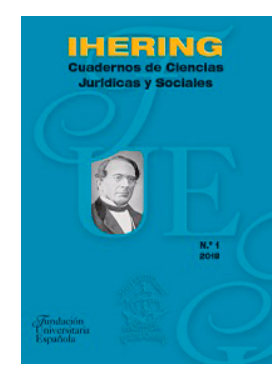

\author{
Ihering. Cuadernos de Ciencias Jurídicas y Sociales \\ $\mathrm{N}^{\mathrm{o}} 1$
}

Año: 2018

e-issn: 2660-552X

DOI: https://doi.org/10.51743/ihering. 8

(c) $($ i) $(9)$

\title{
La creación de una nueva organización internacional: la Comunidad Europea del Carbón y del Acero
}

\author{
MANUELA FERNÁNDEZ RODRÍGUEZ \\ Universidad Rey Juan Carlos
}

\section{1.- PRIMEROS PASOS Y NEGOCIACIÓN DEL TRATADO}

Ta creación de la Comunidad Europea del Carbón y del Acero -en ade-

Llante CECA- representa uno de los logros más importantes de la política estadounidense favorable a la cooperación e integración de los países europeos. De aquella política el exponente más relevante de la década de los cuarenta del siglo XX había sido el Plan Marshall y la consiguiente creación de la Organización Europea de Cooperación Económica. Así, la apertura de un proceso de negociación en Europa, a principios de los años cincuenta, satisfacía la voluntad de Washington de que los países del viejo continente cooperaran entre ellos. Esto es para Gillingham el legado más importante que dejó la extinta CECA, antes que la puesta en marcha de un modelo que sirviera de base al resto de Comunidades Europeas ${ }^{1}$.

\footnotetext{
${ }^{1}$ GILLINGHAM, J., "American Monnetism and the European Coal-Steel Community in the Fifties", Journal of European Integration History, 1995, Vol. 1, Number 1., pp. 21-36, pp. 21-22. La idea estadounidense de acercar a Alemania al proceso de unión europea también rigió a la hora de negociar el rearmamento alemán y la Comunidad Europea de Defensa. PRUESSEN, R. W., "Cold War Threats and America's Commitment to the European De-
} 
La propuesta de puesta en común en Europa Occidental de recursos tales como el carbón y el acero, materias esenciales como fuente de energía y como material básico en la industria armamentística respectivamente, fue elaborada por Jean Monnet y su equipo, a iniciativa de Robert Schuman ${ }^{2}$. Esa propuesta adoptó la forma de Declaración de 9 de mayo de 1950 o Plan Schuman y marcó el camino hacia la firma de un tratado que creó una nueva organización internacional:

"[...] Los principios y compromisos esenciales anteriormente expuestos serán objeto de un tratado firmado entre los Estados $[\ldots]^{\prime 3}$.

Además, aquel documento propugnaba un proyecto abierto a otros estados europeos, más allá de los que asintieron en un primer momento a la Declaración.

A la participación en la futura Comunidad los gobiernos belga y holandés reaccionaron de forma reticente, pues el Plan Schuman afectaba a cuestiones relevantes para ambos países, tales como los efectos de la integración en los países de menor tamaño frente a lo que suponía para los de mayor entidad, como Francia o la República Federal de Alemania, con el riesgo de que la CECA abriera el camino a un directorio franco-alemán. También temían los efectos que pudiera tener la futura organización en sus intereses económicos: a Países Bajos le preocupaba que la cesión de soberanía y el proceso de armonización implícito en la puesta en común de la producción del carbón y el acero produjera una subida de precios y salarios que interfiriera en las políti-

fense Community: One Corner of a Triangle”, Journal or European Integration History, 1996, vol. 1, number 1, pp. 51-69, p. 60.

${ }^{2}$ Monnet dedicó mucho tiempo a desarrollar sus doctrinas sobre los asuntos europeos entre 1950 y 1970. SU, H., "Jean Monnet's Grand Design for Europe and its Criticism”, Journal of European Integration History, 2009, vol. 15, number 2, pp. 29-45, p. 25. Sobre Jean Monnet puede verse además MONNET, J., Mémoirs, Nueva York, 1978. PÉREZBUSTAMANTE, R., Jean Monnet. Ciudadano de Europa, Madrid, 2008. SAN MIGUEL PÉREZ, E., La impaciencia del bien, Madrid, 2015. SÁIZ, J. M., "La vision cristiana de los padres de Europa”, UNISCI Discussion Papers, n 14, mayo 2007, pp. 115-129.

${ }^{3}$ Declaración de 9 de mayo de 1950, puede consultarse en multitud de páginas, como en la de la fundación Robert Schuman:

hps://www.robert-schuman.eu/es/doc/questions-d-europe/qe-204-es.pdf 
cas holandesas de bajos salarios, reduciendo la competitividad de la economía exportadora holandesa, en proceso de reconstrucción tras la guerra. Bélgica, por su parte, siendo un exportador tradicional de acero con un amplio e influyente sector acerero, aunque con altos costes de producción, temía una bajada de salarios y precios, pues el carbón belga, aún con un precio elevado, dejaba escasos beneficios ${ }^{4}$.

Otra de las preocupaciones que despertaba el proyecto era las consecuencias de la no participación británica y, vinculado a ello, el temor a que la participación belga y holandesa supusiera una desvinculación de sus lazos atlánticos a medio y largo plazo ${ }^{5}$. Respecto a esto último, tanto Robert Schuman como Jean Monnet tenían el convencimiento de que Reino Unido participaría en la futura Comunidad, pues su creación se antojaba beneficiosa tanto para las relaciones pacíficas entre estados europeos como desde un punto de vista económico. En cualquier caso, ambas personalidades dejaron claro que el posible rechazo británico no impediría que los países continentales caminaran hacia la integración europea, como de hecho ocurrió, pues Reino Unido no se incorporó a las Comunidades Europeas hasta 1973.

A pesar de los temores y dudas, para Bélgica y Holanda no había más opción que participar de la integración ${ }^{6}$. No era factible rechazar el plan dada la dependencia económica de ambos países de sus vecinos. Sin embargo, junto a Luxemburgo, reaccionaron con escepticismo frente al punto más novedoso de la Declaración de 9 de mayo de 1950: la creación de una institución, denominada Alta Autoridad, destinada a ser la institución principal de la nueva comunidad. Lo que despertaba sus recelos era su carácter supranacional, ya que en ella los países participantes de la Comunidad delegarían su soberanía política respecto al sector del carbón y el acero ${ }^{7}$ convirtiendo a la CECA en una organización supranacional, tal y como se propugnaba en la Declaración de 9 de mayo de 1950. Esa consideración de organismo supranacional es

\footnotetext{
${ }^{4}$ NASRA, S., y SERGES, M., "Between Charlemagne and Atlantis...”, pp. 188-189.

${ }^{5}$ NASRA, S., y SERGES, M., "Between Charlemagne and Atlantis...", p. 186.

${ }^{6}$ En este sentido puede verse HARRYVAN, A. G., In pursuit of influence. The Netherlands European Policy during the Formative Years of the European Union, 1952-1973, Bruselas, 2009.

${ }^{7}$ NASRA, S., y SERGES, M., "Between Charlemagne and Atlantis...”, p. 186.
} 
habitual para la mayoría de los especialistas, salvo excepciones como la de Gillingham, que habla de "la farsa de la supranacionalidad", que explicaría por qué la noción de supranacionalidad se abandonó durante las negociaciones que se llevaron a cabo, a partir de mayo de 1955, para la firma de los Tratados de Roma ${ }^{8}$.

Aunque el proyecto CECA salió adelante, hubo de vencer resistencias. Respecto a los países del Benelux fueron los Países Bajos los que tuvieron un papel más activo en contra de la CECA $^{9}$. El gobierno de Drees-Van Schaik lanzó una contrapropuesta al plan Schuman, como a través del ministro de Asuntos Exteriores, el liberal Dirk Stikker, que proponía una liberalización por sectores dentro de los países integrantes de la Organización Europea de Cooperación Económica, incluyendo así a Reino Unido. Sin embargo, la estricta naturaleza intergubernamental de esta iniciativa quedó ensombrecida por la propuesta integradora del Plan Schuman. Por su parte, la postura defendida por el ministro de Asuntos Exteriores belga, Paul van Zeeland, se centró en defender la soberanía estatal frente al intervencionismo y la pérdida de soberanía, pero más allá de la élite política, las negociaciones para la creación de la CECA no tuvieron gran eco en la opinión pública belga, pues el debate popular estaba protagonizado en ese momento por la sucesión del rey Leopoldo III en su hijo Balduino ${ }^{10}$.

Finalmente, los representantes de los tres países del Benelux -Dirk Stikker, ministro de Asuntos Exteriores de los Países Bajos, Paul Van Zeeland, ministro de Asuntos Exteriores y de Comercio Exterior belga, y Joseph Bech, Primer Ministro de Luxemburgo- aceptaron formar parte del proyecto. Aun así, Bruselas y La Haya unieron fuerzas para presionar a favor del esta-

\footnotetext{
${ }^{8}$ GILLINGHAM, J., “American Monnetism and ...”, pp. 21-22.

${ }^{9}$ Poseían además, en especial Holanda y Bélgica, una tradición jurídica común que se remontaban al periodo de dominación de los Habsburgo. Sobre algunos aspectos legales de ese periodo que dejaron honda huella en la mentalidad de Flandes y los Países Bajos puede verse MARTÍNEZ PEÑAS, L., "La legislación de Carlos V contra la herejía en los Países Bajos", en Revista de la Inquisición (Intolerancia y Derechos Humanos), nº 16 (2012) y "Las dificultades en la persecución de la herejía en Flandes: el caso de Brujas (1564-1565)", en Revista de Historia de la Inquisición (Intolerancia y Derechos Humanos), $\mathrm{n}^{\circ} 18$ (2014).

${ }^{10}$ NASRA, S., y SERGES, M., "Between Charlemagne and Atlantis...”, pp. 187-188.
} 
blecimiento de un Consejo de Ministros intergubernamental como contrapeso a la prevista Alta Autoridad de la CECA ${ }^{11}$.

Francia aceptó la sugerencia de crear un Consejo de Ministros y una Asamblea Común que ampliaran el marco institucional de la futura organización $^{12}$. De esta forma, se presentó un memorándum sobre el funcionamiento de las instituciones en el que, aunque se mantenía el carácter principal de la Alta Autoridad, se establecía que el Consejo de Ministros actuaría como un órgano de relación entre la Alta Autoridad y los gobiernos nacionales, mientras que la Asamblea serviría para controlar a aquella ${ }^{13}$.

Italia, por su parte, también aceptó la invitación a adherirse al proyecto de integración, aunque para ello exigió ocupar un lugar y un papel semejante al de los países que llevaban la iniciativa, es decir, Francia y la República Federal de Alemania. Una de las peticiones italianas durante las negociaciones fue que los territorios de ultramar franceses fueran incluidos en la CECA, a lo que Jean Monnet respondió con recelo, puesto que requeriría una modificación del sistema de Unión Francesa. Los territorios de ultramar no fueron incluidos finalmente en la CECA, pero Francia fue obligada a conceder al resto de estados miembros las mismas medidas preferenciales de las que disfrutaba el país galo ${ }^{14}$.

${ }^{11}$ NASRA, S., y SERGES, M., "Between Charlemagne and Atlantis...”, p. 188.

${ }^{12} \mathrm{Ni}$ la Asamblea ni el Consejo de Ministros formaron parte de la estructura institucional diseñada por los autores del Plan Schuman. Sí había un Tribunal de Justicia o al menos un organismo independiente de control que vigilara y se asegurara de la legalidad de las actuaciones de la Alta Autoridad. PENNERA, C., "The Beginnings of the Court of Justice and its Role as driving Force in European Integration", Journal of European Integration History, 1995, Vol. 1, Number 1., pp. 111-127, p. 112.

${ }^{13}$ NASRA, S., y SERGES, M., "Between Charlemagne and Atlantis...”, p. 189. Las empresas siderúrgicas francesas mayoritariamente aprobaron el contenido del Plan Schuman, salvo el texto definitivo de los artículos 65 y 66 del tratado, relativos a la prohibición de cualquier forma de asociación o acuerdos entre empresas que pudieran tener como resultado una limitación de la libre competencia establecida en el mercado común del carbón y acero, algunos acuerdos podrían llevarse a cabo si la Alta Autoridad así lo acordase, también en ellos se recogía la posibilidad de que la Alta Autoridad impusiera sanciones pecuniarias. BERGER, F., "Les sidérurgistes français et allemands face à l'Europe: convergences et divergences de conception et d'intérêts 1932-1952", Journal of European Integration History, 1997, Vol. 3, Number 2, pp. 35-52, pp. 49-50.

${ }^{14}$ La Unión Francesa estaba formada por la Francia metropolitana, los departamentos de 
El intento de implicar a Reino Unido tuvo un resultado diferente. A finales de mayo de 1950 se envió a Londres un memorándum solicitando su participación en el proyecto pero la invitación fue rechazada, dado que, en ese momento, los británicos no estaban dispuestos a formar parte de una organización que tuviera como rasgo principal la creación de una institución como la Alta Autoridad, a la que se le preveían poderes supranacionales ${ }^{15}$. El 2 de junio así lo hizo saber ${ }^{16}$; sin embargo su no participación no fue pasiva, sino más bien lo contrario: los británicos intentaron interferir en la idea de la Comunidad Europea del Carbón y del Acero proponiendo una forma de organización vinculada al Consejo de Europa - igual que hizo años después cuando se negociaba el Tratado de la Comunidad Económica Europea, proponiendo en esta ocasión el establecimiento de una zona de libre cambio a los países miembros de la OECE $-{ }^{17}$. La propuesta no contó con el respaldo

ultramar (los departamentos argelinos, Martinica, las islas de La Reunión, Guadalupe y la Guyana Francesa), los territorios de ultramar (antiguas colonias francesas), los territorios asociados (Camerún y Togo administrados bajo la supervisión del Consejo de Naciones Unidas) y los Estados Asociados (los protectorados de Marruecos, Túnez e Indochina).KIM, S. R., "France's Agony between "Vocation Européenne et Mondiale". The Union Française as an Obstacle in the French Policy of Supranational European Integration, 19521954", Journal of European Integration History, 2002, vol. 8, number 1, pp. 61-84, p. 6366. En aquellos años, España aun retenía el Protectorado de Marruecos, sobre cuya administración puede leerse MARTÍNEZ PEÑAS, L., "La administración central del Protectorado, 1912-1936”, en ALVARADO PLANAS, J., y DOMÍNGUEZ NAFRÍA, J. C., La Administración del Protectorado de Marruecos. Madrid, 2014.

${ }^{15}$ En aquellos años, Reino Unido afrontaba una oleada de conflictos armados en sus colonias y dominios, respecto de la cual puede verse MARTÍNEZ PEÑAS, L., En nombre de su Majestad. Valladolid, 2016; el principal de esos conflictos fue el conflicto norirlandés, respecto del cual puede verse el artículo de Erika Prado al respecto en VV.AA, El año de los doce mayos. Valladolid, 2018.

${ }^{16}$ LORD, C. "With but not of". Britain and the Schuman Plan, a reinterpretation", Journal of European Integration History, 1998, Vol. 4, Number 2., pp. 23-46, pp. 25-26. Este autor considera que; a pesar del rechazo británico en ese momento, el gobierno siempre barajó la posibilidad de adherirse o asociarse con posterioridad. No en vano, en el año 1954, se firmó un acuerdo de asociación entre Reino Unido y la Comunidad del Carbón y Acero.

17 También en la creación de la Comunidad Europea del Carbón y del Acero Reino Unido tuvo una actitud reticente a formar parte de la integración sectorial. Sobre las fricciones entre la Francia de De Gaulle e Inglaterra en relación con su adhesión a las Comunidades Europeas puede verse MENÉNDEZ, J., "Francia, Ingla- 
de otros estados europeos y Francia manifestó su rechazo con meridiana claridad $^{18}$.

De este modo, se concluyó un acuerdo previo entre los que se convirtieron en los seis Estados fundadores de las Comunidades Europeas, Unión Europea en la actualidad: República Federal de Alemania, Francia, Italia, Bélgica, Países Bajos y Luxemburgo, superando así el núcleo franco-alemán la rivalidad secular que había existido entre ambos países.

Las negociaciones, organizadas por Jean Monnet, comenzaron el 20 de junio de 1950. Él mismo presentó un proyecto de tratado formado por cuarenta artículos, tomando como documento base la Declaración de 9 de mayo. La creación de la CECA vio su futuro peligrar con ocasión del estallido de la guerra de Corea, el 25 de junio de 1950, debido a que en septiembre de ese mismo año Estados Unidos propuso permitir el rearme de Alemania Occidental, surgiendo así la idea, paralela a la Comunidad Europea del Carbón y del Acero, de formar un ejército europeo compuesto por unidades nacionales -lo que terminaría siendo la Comunidad Europea de Defensa- ${ }^{19}$.

Entre septiembre y octubre de 1950 se realizó la primera redacción del proyecto de tratado que quedaría acabado el 9 de noviembre, dando salida a cuestiones de carácter político como la posición del Sarre y el futuro del

terra y el Mercado Común”, Revista de Política Internacional, no 95, 1968, pp. 3960. También MERCADAL, J., "Inglaterra y el Mercado Común”, pp. 21-41.

${ }^{18}$ LARRES, K., "Integrating Europe or Ending the Cold War? Churchill's post-war foreign policy", Journal or European Integration History, 1996, vol. 1, number 1, pp. 15-49, p. 34.

${ }^{19}$ GILLINGHAM, J., “American Monnetism and ...”, p. 25. Sobre los fracasos de creación de una Comunidad Europea de Defensa y una Comunidad Política Europea puede verse LAURENT, M., "Los proyectos de integración europea entre 1954 y la conferencia de Messina de junio de 1955", Historia crítica, no 16, 1998. BOSSUAT, G., Histoire de l'Union européenne. Fondations, élargissements, avenir. Belin, 2009, p. 165. TRACHTENBERG, M., y GEHRZ, C., "America, Europe and German Rearmament, August-Septembre 1950", Journal or European Integration History, 2000, vol. 6, number, 2, pp. 9-35, p. 9. RISSO, L., "Against Rearmament or Against Integration? The PCI and PCF's Opposition to the European Defense Community and the Western European Union, 1950-1955", Journal of European Integration History, 2007, vol. 13, number 2, pp. 11-31, p. 26. La guerra fue un factor clave en el desarrollo supraestatal de Europa, de la misma forma en que lo había sido antes del desarrollo estatal, tal y como puede verse en MARTÍNEZ PEÑAS, L., y FERNÁNDEZ RODRÍGUEZ, M., La guerra y el nacimiento del Estado Moderno. Valladolid, 2014. 
estatuto del Ruhr. En nombre del principio de igualdad, el canciller Adenauer, el Secretario de Estado de Asuntos Exteriores, Hallstein y los industriales del Ruhr reclamaron el fin de las prohibiciones y de las obligaciones que pesaban sobre Alemania como consecuencia de su actuación en la Segunda Guerra Mundial. El gobierno francés informó al canciller Adenauer que emprendería ante Estados Unidos y Reino Unido, potencias ocupantes, junto con Francia, de Alemania Occidental, las gestiones necesarias para la supresión de los controles de la industria carbonera y siderúrgica que fueran contrarios a las cláusulas del Tratado. De esta forma, la Autoridad Internacional del Ruhr puesta en marcha en mayo de 1949 quedó superada por el tratado CECA, produciéndose su disolución en febrero de 1953.

Entre marzo y abril de 1951 tuvieron lugar las últimas reuniones de las seis delegaciones en las que se afrontaron cuestiones como el número de miembros de la Alta Autoridad y la forma de su designación, la composición y las modalidades del voto en el Consejo de Ministros y el número de representantes de los países en la Asamblea. Además, se propuso una representación igualitaria para Francia y la República Federal de Alemania, que debía mantenerse en el desarrollo ulterior de la organización europea. Todo ello permitió finalizar el tratado y los acuerdos anexos.

Las negociaciones del tratado del carbón y acero provocaron, como no podía ser de otro modo, reacciones más allá de los seis países firmantes. Por ejemplo, la Unión Soviética manifestaba que la negociación al respecto del futuro tratado era una violación de los acuerdos de Postdam de agosto de 1945, pues argumentaba que en aquellos los aliados se comprometían a no ayudar económicamente a la reconstrucción de la República Federal de Alemania $^{20}$. Además, se identificaba a la CECA con otros intentos imperialistas de unificar Europa, incluyendo el de Hitler. Estas afirmaciones fueron

${ }^{20}$ A este respecto puede verse MARTÍNEZ PEÑAS, L., Introducción a la historia de las relaciones internacionales, Valladolid, 2016, pp. 119-120. De este mismo autor, relacionado con la cuestión puede verse Sic transit gloria mundi, Valladolid, 2017. De forma genérica respecto a las Relaciones Internacionales en la antigüedad: Y lo llamarán paz. Visión jurídico-institucional de las relaciones internacionales en la Antigüedad, Valladolid, 2018; y en el medievo: El invierno. Visión jurídico-institucional de las relaciones internacionales en la Antigüedad. Valladolid, 2019, actualmente en prensa. 
una constante durante las negociaciones tanto del tratado -CECA- como el posterior de la Comunidad Europea de Defensa -CCD- Las protestas soviéticas no hicieron peligrar ninguno de los proyectos de integración occidental aunque el bloque oriental llegó a ofrecer una cooperación europea más amplia que la de los países del Occidente europeo, de modo que tanto la CECA como la CED parecieran superfluas. Con esta finalidad, durante el proceso de ratificación de la CECA, se convocó una conferencia económica internacional que tuvo lugar en Moscú, del 3 al 12 de abril de 1952, para desarrollar el comercio entre el Este y el Oeste. La reunión, boicoteada por los gobiernos occidentales, consiguió reunir 450 asistentes de 47 países y en ella se solicitó a las Naciones Unidas que organizara una conferencia sobre comercio abierta a toda Europa y descartara proyectos supranacionales sobre el comercio de bienes esenciales $^{21}$.

Finalmente, el Tratado y sus anexos serían firmados en París el 18 de abril de 1951. La ratificación se desarrolló entre junio de 1951 y junio de 1952 siguiendo lo previsto en el artículo 99 que preveía que el tratado entrara en vigor el día en que se depositara el instrumento de ratificación del último estado firmante. Esto tuvo lugar el 24 de julio de 1952, después de que Italia, último país que votó la ratificación, entregase el instrumento de ratificación al gobierno francés, depositario del Tratado.

\section{2.- OBJETIVOS, FUNCIONAMIENTO Y CONTROVERSIA RESPECTO A LA COMPOSICIÓN INSTITUCIONAL}

El Tratado constitutivo de la Comunidad Europea del Carbón y del Acero estaba formado por un preámbulo, inspirado en la Declaración de 9 de mayo de 1950, cien artículos y tres anexos en los que se definían los materiales a los que se aplicaba el contenido del tratado: carbón, acero, chatarra y aceros especiales. Además, se añadían varios protocolos que afectaban a distintas materias: un protocolo sobre los privilegios e inmunidades de la Comunidad,

${ }^{21}$ MUELLER, W., "The Soviet Union and Early West European Integration, 1947-1957: From the Brussels Treaty to the ECSC and the EEC", Journal of European Integration History, 2009 Vol. 15, Number 2., pp. 67-85, pp. 74-81. 
otro sobre el estatuto del Tribunal de Justicia, un tercero sobre las relaciones con el Consejo de Europa, el canje de cartas entre el gobierno alemán y el francés acerca del estatuto del Sarre y un convenio relativo a las disposiciones transitorias.

El título primero contiene seis artículos que, bajo la denominación de "La Comunidad Europea del Carbón y del Acero", establecen los elementos esenciales de la Comunidad: un mercado común con objetivos comunes e instituciones comunes ${ }^{22}$. Los objetivos se enumeraban en los artículos 2 y 3 , encargando a las instituciones velar por el abastecimiento regular del mercado común, teniendo en cuenta las necesidades de terceros países; asegurar a los usuarios que se encuentren en condiciones comparables igual acceso a las fuentes de producción; velar por la fijación de precios lo más bajos posibles; velar por el mantenimiento de condiciones que estimulen a las empresas a desarrollar y mejorar su capacidad de producción y a promover una política de explotación racional de los recursos naturales; promover la mejora de las condiciones de vida y de trabajo de la mano de obra; fomentar el desarrollo de los intercambios internacionales; promover la expansión regular y la modernización de la producción, así como la mejora de la calidad, en condiciones tales que descarten toda protección frente a las industrias competidoras.

En el artículo 4 TCECA se recogían las prácticas incompatibles con el concepto de mercado común y, por tanto, prohibidas, como eran los derechos de salida o entrada, las exacciones de efecto equivalente, las restricciones cuantitativas a la circulación de los productos; las medidas o prácticas discriminatorias entre productores, entre compradores o entre usuarios, especialmente en lo concerniente a las condiciones de precios o de entrega y a las tarifas de transporte; las medidas o prácticas que obstaculizaran la libre elección por el comprador de su abastecedor; las subvenciones o ayudas otorgadas por los Estados o los gravámenes especiales impuestos por ellos, cualquiera que sea su forma y las prácticas restrictivas tendentes al reparto de los mercados.

En el título segundo, artículos 7 a 45, se regulaban las instituciones de la organización. Una Alta Autoridad, asistida por un Comité Consultivo, una Asamblea Común, un Consejo Especial de Ministros y un Tribunal de Justi-

${ }^{22}$ Artículo 1 TCECA. 
cia. El propio texto las enumeraba de forma abreviada: Asamblea, Consejo y Tribunal.

La Alta Autoridad -artículos 8 a 19 TCECA- fue la institución principal, por ello se le otorgó la función de asegurar la consecución de los objetivos previstos en el tratado y se convirtió en el principal organismo decisor. Así se establecía en el artículo 14:

"Para el cumplimiento de la misión a ella confiada, la Alta Autoridad tomará decisiones, formulará recomendaciones o emitirá dictámenes en las condiciones previstas en el presente tratado. Las decisiones serán obligatorias en todos sus elementos. Las recomendaciones obligarán en cuanto a los objetivos fijados en ellas, pero dejarán a sus destinatarios la elección de los medios apropiados para alcanzar esos objetivos. Los dictámenes no serán vinculantes $[\ldots]^{\prime 23}$.

La Alta Autoridad emitía opiniones, hacía recomendaciones y tomaba decisiones, aunque para algunas de ellas era necesario dictamen conforme del Consejo de Ministros. En opinión de Bitsch, así se establecían entre Consejo y Alta Autoridad relaciones de distinto tipo en función del momento o la decisión: cooperación-rivalidad-enfrentamiento ${ }^{24}$.

La composición de la Alta Autoridad fue problemática debido a su carácter supranacional. Se buscaba asegurar la independencia y la competencia técnica de sus miembros. La elección del número de sus integrantes tampoco fue sencilla. La Declaración de 9 de mayo de 1950 partía de la premisa de que los miembros de la Alta Autoridad serían designados sobre una base paritaria buscando reflejar la igualdad entre Francia y la República Federal, naciones marcadas por el enfrentamiento mutuo, pero no aspiraba a que todos los Estados tuvieran el mismo número de miembros. Se barajaron distintas posibilidades, ganando adeptos la que preveía que el número de miembros de la institución fuera de seis a nueve, pues ambos números eran múlti-

${ }^{23}$ Este poder decisorio lo ejercía de forma autónoma en la mayor parte de los casos, o con opinión conforme del Consejo, en aquellas ocasiones que así lo preveía el texto. LAPIÉ, Las tres Comunidades Europeas... pp. 55-56.

${ }^{24}$ BITSCH, M.T., "La premiére institution supranationales: du nouveau sur l'histoire de la Haute Autorité de la Communauté européenne du charbo et de l'acier", Journal of European Integration History, 1995, Vol. 1, Number 1., pp. 129-141, p. 133. 
plos de tres, lo que permitiría obtener fácilmente una mayoría de dos tercios y contentar a los países de menor tamaño, menor demografía y menor peso económico -Bélgica, Holanda y Luxemburgo-, que con tales cifras, iguales o superiores al número de estados miembros de la futura Comunidad, aspiraban a tener a un nacional propio en la composición de la Alta Autoridad, algo imposible si el número de componentes fuera inferior al número de estados. Era mucho más fácil aceptar la independencia de la institución sabiendo que en ella podría haber, al menos, un nacional de cada Estado miembro ${ }^{25}$.

La delegación francesa propugnaba la mayor eficacia de la institución en cuanto a la toma de decisiones, cuanto menor fuera el número de miembros. Con la cifra de cinco miembros era más evidente el carácter supranacional de la institución, pues si esta velaba por el interés común y sus miembros debían ser independientes de aquellos estados de los que eran nacionales no había necesidad de que todos los países contaran con representación. Para los tres países de menor tamaño este planteamiento era inconcebible, especialmente para Luxemburgo que, por sus dimensiones, era consciente de que sería el país que quedaría sin representación. La postura luxemburguesa defendía una composición de nueve miembros, dos para Francia y Alemania, uno para cada uno de los tres miembros del Benelux, otro para Italia y un noveno de carácter rotario. Otra propuesta, lanzada por Países Bajos, fue la de siete miembros. Las bondades de esta cifra eran que era impar - lo que evitaría los empates-, que permitía que cada Estado tuviera un representante siendo el séptimo un miembro de las organizaciones sindicales relevantes en el sector objeto del tratado y que era un número lo bastante reducido como para que los acuerdos resultaran ágiles ${ }^{26}$.

En marzo de 1951, apenas un mes antes de la firma del tratado, la cuestión relativa al número de miembros de la Alta Autoridad estaba todavía en el aire. El acuerdo solo parecía posible si todos los países acababan teniendo representación, pues de otra forma no se alcanzaría el consenso. Esta cues-

\footnotetext{
${ }^{25}$ BOERGER-DE SMEDT, A., "Derrière la valse de chiffres. Retour sur les origines de la composition de l'exécutif européen", Journal of European Integration History, 2007, vol. 13, number 1, pp. 25-48, pp. 27-29.

${ }^{26}$ BOERGER-DE SMEDT, A., "Derrière la valse de chiffres...”, pp. 30- 32.
} 
tión no fue sólo una preocupación de los Estados de menor tamaño, sino que Italia también condicionó su apoyo al carácter supranacional de la organización siempre que el país tuviera un representante. De igual modo ocurría con Alemania, para la cual era necesario que los países mayores productores de las materias objeto del tratado pudieran tener un mayor peso representativo, de modo que, países de menor importancia económica no pudieran imponer decisiones desfavorables a los intereses de los grandes productores, de ahí que Ludwig Erhard, el ministro de economía alemán, fuera partidario de la ponderación de los representantes, atendiendo al peso de las industrias, más que a un reparto por países ${ }^{27}$.

Dados los constantes desacuerdos sobre la cuestión, la composición de la Alta Autoridad y el procedimiento de designación de sus miembros se dejaron para un momento final de las negociaciones, a fin de que fuera resuelto a nivel de los ministros de Asuntos Exteriores, en abril de 1951. El 15 de abril se alcanzó el consenso: siete miembros, un representante por Estado y el séptimo cooptado. Estableciéndose que, tras un período transitorio de seis años, se reducirían los representantes a cinco de designación común por los gobiernos de los Estados miembros. En este último caso no podría haber más de dos miembros con la misma nacionalidad. Sin embargo, la reducción tras el período transitorio no se aceptó salvo que la modificación se tomara posteriormente en el Consejo de Ministros por unanimidad, es decir, pasados los seis años la decisión volvería a depender de aquellos mismos que en este momento no eran capaces de ponerse de acuerdo. Esto llevaría a que, en la práctica, la institución contara con nueve miembros ${ }^{28}$.

El resultado fue una institución formada por demasiados miembros, en opinión de Francia, pero sobre la que se podría volver a negociar más tarde. No se estableció ningún plazo, sino que la modificación del número de miembros podría acordarse por unanimidad en el Consejo Especial de Ministros en cualquier momento $^{29}$. En el texto del Tratado no se hacía mención expresa a que cada país miembro debiera tener representación en la Alta Autoridad, pero la composición

\footnotetext{
${ }^{27}$ BOERGER-DE SMEDT, A., "Derrière la valse de chiffres...”, pp. 30- 32.

${ }^{28}$ BOERGER-DE SMEDT, A., "Derrière la valse de chiffres...”, pp. 33- 34.

${ }^{29}$ Esta previsión también se adoptó para otras instituciones, como es el caso del Tribunal de Justicia.
} 
numérica, así como el procedimiento de designación de los miembros prácticamente lo aseguraba y así lo ha demostrado el paso del tiempo, no sólo para la Alta Autoridad, sino para la posterior Comisión Europea, heredera de aquella, actualmente formada por un nacional por cada Estado -veintiocho miembros-, aunque los comisarios están obligados a representar los intereses de la Unión Europea en su conjunto.

El texto establecía que los miembros de la Alta Autoridad no representarían ni los intereses de sus gobiernos ni de los medios industriales, sino que velarían por los objetivos comunes establecidos en el tratado. Para ello, no eran elegidos en función de su nacionalidad, sino por competencia general. No era sencillo, lógicamente, independizar a los miembros de la Alta Autoridad de la representación de los Estados de los que eran nacionales y la negociación del número de miembros así lo puso de manifiesto. El método de designación, que dependía de los gobiernos de los Estados miembros, favorecía la presencia de un nacional por cada Estado miembro sin necesidad de consignar el principio de representación nacional de forma expresa. Además, al no recogerse ese principio la institución podía mantener su aspecto de institución supranacional. Lo que sí especificó el tratado es que sólo nacionales de los países miembros podrían ser representantes de la Alta Autoridad. En cuanto al procedimiento de designación, el artículo 10 TCECA establecía que los gobiernos nombrarían de común acuerdo a ocho miembros y el noveno sería cooptado, necesitando reunir cinco votos de los ocho posibles. Durante los seis primeros años de vida de la organización, la designación de los miembros de la Alta Autoridad requería unanimidad de los Estados miembros, pasado ese plazo, era suficiente una mayoría de cinco sextos. Los gobiernos gozaban de un derecho de veto frente a los candidatos, aunque si se hiciera un uso abusivo del mismo, dos personas en caso de renovación parcial y cuatro en el caso de renovación general, cualquier Estado podría llevar a otro ante el Tribunal de Justicia para que declarara nulo el veto ${ }^{30}$.

De entre los nueve miembros de la institución, uno ejercía la función de presidente de la misma, encargado de la administración de los servicios y de

${ }^{30}$ BOERGER-DE SMEDT, A., "Derrière la valse de chiffres...”, pp. 27- 33. 
asegurar la ejecución de las deliberaciones ${ }^{31}$. También había un vicepresidente y ambos cargos, presidente y vicepresidente, desempeñaban su tarea durante dos años, designados por los gobiernos de los Estados miembros de común acuerdo. Hasta la fusión de la Alta Autoridad con las Comisiones de la Comunidad Económica Europea y la de la Comunidad Europea de la Energía Atómica en 1967, momento en el que desapareció la denominación de Alta Autoridad, los presidentes de la misma fueron Jean Monnet de 1952 a1955 $5^{32}$, René Mayer de 1955 a 1957, Paul Finet durante 1958 y1959, Piero Malvestiti, de 1959 a 1963 y, por último, Dino Del Bo de 1963 a $1967^{33}$.

Respecto a la duración del mandato de los miembros, se les designaba por seis años renovables. Además de por la finalización del mandato, este podía expirar por renovación regular, -cada dos años se procedía a la renovación de un tercio de sus miembros-, por prosperar una moción de censura que implicaba la dimisión colectiva de todo el colegio de comisarios-, por fallecimiento o por dimisión ${ }^{34}$.

Los miembros de la Alta Autoridad debían ejercer sus funciones con plena independencia y en el interés general de la Comunidad, dado el carácter supranacional de la institución. En este sentido, el artículo 9 TCECA ordenaba que:

"en el cumplimiento de sus deberes, no soliciten ni acepten instrucciones de ningún gobierno ni de ningún organismo. Se abstendrán de realizar cualquier acto incompatible con el carácter supranacional de sus funciones".

Un límite semejante se daba en el mismo artículo a los Estados: “cada Estado miembro se obliga a respetar este carácter supranacional y a no intentar

${ }^{31}$ LAPIÉ, Las tres Comunidades Europeas... pág. 52.

${ }^{32}$ Se considera de forma generalizada que Jean Monnet dejó el cargo como presidente de la Alta Autoridad debido al fracaso de la CED. Sin embargo, Gilligham plantea que también podía haberle influido el menos espectacular fracaso de la CECA. GILLINGHAM, J., “American Monnetism and...", pp. 21-36, p. 29.

${ }^{33}$ BITSCH, M.T., "La premiére institution supranationales: du nouveau sur l'histoire de la Haute Autorité de la Communauté européenne du charbo et de l'acier", Journal of European Integration History, 1995, Vol. 1, Number 1., pp. 129-141, p. 129.

${ }^{34}$ LAPIÉ, Las tres Comunidades Europeas... p. 53. 
influir en los miembros de la Alta Autoridad en la ejecución de sus tareas". Los miembros de la Alta Autoridad no podían ejercer ninguna otra actividad profesional, remunerada o no, ni adquirir ni conservar ningún interés que estuviera directa o indirectamente relacionado con los sectores del carbón y del acero, ni durante el tiempo de su mandato, ni tres años después de haber finalizado el mismo ${ }^{35}$. Respecto a su funcionamiento, para preparar las deliberaciones, como muchos órganos colegiados, sus miembros formaban grupos de trabajo que se encargaba de elaborar las propuestas. Las deliberaciones se adoptaban por mayoría de los miembros que la componían ${ }^{36}$, sometidas a un quórum de cinco. Las sesiones de la Alta Autoridad eran reservadas, aunque se levantaba un acta de las mismas ${ }^{37}$.

Como estipulaba el artículo 7 TCECA, la Alta Autoridad estaba asistida en sus funciones por un órgano de consulta, denominado Comité Consultivo, previsto en los artículos 18 y 19 del Tratado. Este organismo estaba formado por no menos de treinta y no más de cincuenta y un miembros, de entre productores, trabajadores, usuarios y comerciantes, comprendiendo un número igual cada grupo. Su designación correspondía al Consejo, quien además designaba a las organizaciones representativas entre las que se distribuirán los puestos por un período de dos años.

Otra de las instituciones de la Comunidad era la Asamblea regulada en los artículos 20 a 25 TCECA. Según estos, la Asamblea estaba formada por "representantes de los pueblos de los Estados reunidos en la Comunidad". De esta forma, cada año, se designaban delegados de los parlamentos nacionales. El Tratado preveía la posible elección de sus miembros por sufragio universal directo, atribuyendo a la propia Asamblea la misión de elaborar las propuestas para su implementación. Esta previsión no se cumplió hasta el verano del año 1979. Los delegados se distribuían del siguiente modo: dieciocho miembros para cada uno de los parlamentos de

\footnotetext{
${ }^{35}$ Hace notar Lapié, que es en este artículo, el único lugar en todo el Tratado en que se utiliza la palabra supranacional, con ocasión de tratar del carácter de la función de los miembros. LAPIÉ, Las tres Comunidades Europeas... pp. 51 y 52.

${ }^{36}$ Artículo 13 TCECA.

${ }^{37}$ El quórum no se recoge en el Tratado sino en el Reglamento de aplicación de la Alta Autoridad. LAPIÉ, Las tres Comunidades Europeas... pp. 53-56.
} 
Alemania, Francia e Italia; diez, en el caso de Bélgica y Holanda y cuatro para Luxemburgo, haciendo un total de setenta y ocho miembros ${ }^{38}$. La propia Asamblea designaría de entre sus miembros al presidente de la institución y a la Mesa ${ }^{39}$. A pesar de lo que su denominación pudiera hacer pensar, la Asamblea no tenía poder legislativo, sino que era un órgano que ejercía las competencias de control que se le atribuían a lo largo del Tratado. Manifestaciones de este control eran discutir y aprobar el informe anual de la Alta Autoridad, plantear cuestiones escritas u orales a otras instituciones y censurar a la Alta Autoridad si obtuviese una mayoría de dos tercios de los votos de la mayoría absoluta de los miembros de la Asamblea ${ }^{40}$.

Respecto a su funcionamiento, la Asamblea celebraba cada año un período de sesiones que no podía prolongarse más allá del final del ejercicio económico en curso. Se reunía, sin necesidad de previa convocatoria, el segundo martes de mayo. También podía ser convocada en periodo extraordinario, a petición del Consejo, para emitir un dictamen sobre las cuestiones que éste le sometiera, o a petición de la mayoría de los representantes de la Asamblea o de la Alta Autori$\mathrm{dad}^{41}$. A sus sesiones podían acudir, tanto los miembros de la Alta Autoridad, como del Consejo y podían ser oídos en la misma si así lo solicitaban ${ }^{42}$.

La tercera de las instituciones fue el Consejo, regulado en los artículos 26 a 30 TCECA, que estaba compuesto por los representantes de los Estados miembros a través de un miembro del gobierno. Cada miembro del Consejo ejercía la presidencia del órgano por orden rotatorio durante un período de tres meses, siguiendo el orden alfabético de los nombres de los Estados miembros en su propia lengua ${ }^{43}$. Esta institución tenía como función armonizar la acción de la Alta Autoridad y la de los gobiernos encargados de la política económica general de sus países. Para ello, el Consejo de Ministros y la Alta Autoridad debían proceder al intercambio de informaciones y a

\footnotetext{
${ }^{38}$ Artículo 21.

${ }^{39}$ Artículo 23 TCECA.

${ }^{40}$ BITSCH, M.T., "La premiére institution...," pp. 129-141, p. 133.

${ }^{41}$ Artículo 22 TCECA.

${ }^{42}$ Artículo 23 TCECA.

${ }^{43}$ Artículo 27 TCECA.
} 
consultarse mutuamente, pudiendo solicitar a la Alta Autoridad que procediera al estudio de todas las propuestas y medidas que considerase oportunas o necesarias para la consecución de los objetivos comunes ${ }^{44}$. Respecto a su funcionamiento, el Consejo se reuniría a instancia de su presidente, a petición de un Estado miembro o de la Alta Autoridad. Para la aprobación de un dictamen del Consejo, en aquellos casos en que el Tratado así lo exigiera, se requería la mayoría absoluta de los representantes de los Estados miembros, incluido el voto del representante de uno de los Estados que aseguren al menos el $20 \%$ del valor total de las producciones de carbón y acero de la Comunidad. En caso de igualdad de votos, y si la Alta Autoridad mantuviere su propuesta tras una segunda deliberación se requería el voto de los representantes de dos Estados miembros que asegurasen cada uno de ellos al menos el $20 \%$ del valor total de las producciones de carbón y de acero de la Comunidad. En otras ocasiones se requería que la decisión o dictamen fueran adoptados por unanimidad o por mayoría simple ${ }^{45}$, requiriéndose, respectivamente, el voto de todos los miembros del órgano o el voto de la mayoría absoluta, incluido el voto del representante de uno de los estados que asegurasen al menos el $20 \%$ del valor total de la producción respectivamente.

Por último, el Tribunal de Justicia se encontraba recogido en los artículos 31 a 45 del Tratado. Su función era asegurar el respeto del derecho en la interpretación y la aplicación del texto y de sus reglamentos de ejecución ${ }^{46}$. Así, velaba por la legalidad de las decisiones y actos de la Alta Autoridad, del Consejo y de la Asamblea ${ }^{47}$, a petición de un Estado miembro o de la Alta Autoridad $^{48}$, lo que permitía que el Tribunal pudiera declarar una decisión o recomendación como nula y sin validez ${ }^{49}$. En la propuesta inicial elaborada por Jean Monnet, no se había previsto el establecimiento de un Tribunal de Justicia, pues se temía que su existencia pudiera socavar los pode-

\footnotetext{
${ }^{44}$ Artículo 26 TCECA.

${ }^{45}$ Artículo 28 TCECA.

${ }^{46}$ Artículo 31 TCECA.

${ }^{47}$ RASMUSSEN, M., "The Origins of a Legal Revolution. The Early History of the European Court of Justice", Journal of European Integration History, 2008, Vol. 14, Number 2., pp. 77-98, p. 83.

${ }^{48}$ Artículo 38 TCECA.

${ }^{49}$ Artículos 33 a 35 TCECA.
} 
res de la Alta Autoridad. Originalmente se aspiraba, más bien, a incluir algún tipo de tribunal de arbitraje internacional no permanente, pero bajo la presión de Alemania, Bélgica, Países Bajos y Luxemburgo se creó un tribunal de justicia permanente para dirimir disputas entre los Estados miembros y entre estos y la Alta Autoridad ${ }^{50}$.

Respecto a su composición, el Tribunal estaba formado por siete jueces, designados de común acuerdo por los gobiernos de los Estados miembros, por un período de seis años renovables, entre personalidades que ofrecieran absolutas garantías de independencia y competencia. Los jueces, de entre ellos, designarán al presidente del Tribunal por un período de tres años ${ }^{51}$. Cada tres años tendría lugar una renovación parcial que afectaría alternativamente a tres y cuatro miembros ${ }^{52}$.

La composición del Tribunal de Justicia fue objeto de intensos debates. El número de sus miembros y el modo de designación suscitaron problemas análogos a los de la composición de la Alta Autoridad. El documento de trabajo francés hablaba en un primer momento de cinco jueces: tres elegidos por los Estados miembros y dos nombrados, respectivamente, por la Corte Internacional de Justicia de Naciones Unidas y la Organización Internacional del Trabajo. Por su parte, el gobierno neerlandés sugirió que el Tribunal contara con tantos jueces como Estados miembros y dos más nombrados por la Corte y por la OIT. Alemania, propuso nueve jueces y nueve suplentes elegidos por un período de seis años por la Asamblea Común ${ }^{53}$.

Finalmente, el artículo 32 del Tratado recogió siete jueces, en lugar de los cinco propuestos por Francia, o los ocho o nueve planteados por Países Bajos y Alemania. El número de siete jueces, habiendo seis Estados miembros fundadores de la Comunidad, vino determinado por la necesidad de que adoptara sus decisiones por mayoría y se evitara el empate de

\footnotetext{
${ }^{50}$ Artículo 89 TCECA.

${ }^{51}$ Artículo 32 TCECA.

${ }^{52}$ Artículo 32 TCECA.

${ }^{53}$ BOERGER-DE SMEDT, A., "La Cour de Justice dans les négociations du traité de Paris instituant la CECA", Journal of European Integration History, 2008, Vol. 14, Number 2., pp. 7-33, p. 19.
} 
votos. Se consiguió el consenso dado que siendo un número superior al de los países miembros permitía que cada Estado designara un nacional, aunque esta previsión, igual que en el caso de la Alta Autoridad, no se recogía expresamente en el texto del Tratado $^{54}$, por lo que podrían designarse jueces que no fueran nacionales de ninguno de los Estados miembros de la organización, aunque obviamente, y el tiempo, así lo ha puesto de manifiesto, esto no entraba en las previsiones de los miembros, que no querían perder control de ninguna de las instituciones que formaban parte del proceso de integración europea, en aras de la independencia, imparcialidad o supranacionalidad. La composición del primer Tribunal ya lo puso de manifiesto, cada Estado envió un juez a Luxemburgo y el séptimo fue un sindicalista, los dos abogados generales fueron francés y alemán (Lagrange y Karl Roemer) y, para satisfacer las reivindicaciones de Roma de tener una relevancia semejante a Francia y la República Federal alemana, el juez italiano Massimo Pilotti, presidió el órgano ${ }^{55}$. El Tribunal, que estableció su sede, al igual que la Alta Autoridad, en Luxemburgo, comenzó a funcionar el 4 de diciembre de 1952, cuando sus miembros juraron el cargo ${ }^{56}$. La sede de las instituciones, igual que la composición de la Alta Autoridad y el Tribunal de Justicia también fue una cuestión comprometida. Las negociaciones fueron difíciles, hasta tal punto que no se recogió esta cuestión en el texto del Tratado ${ }^{57}$. De forma, en principio provisional, a instancias del primer ministro de Luxemburgo se instalaron en este país, salvo la Asamblea, que compartió espacio con la Asamblea del Consejo de Europa en Estrasburgo ${ }^{58}$.

\footnotetext{
${ }^{54}$ PENNERA, C., "The Beginnings of the Court of Justice ...”, pp. 115-116. En opinión del autor, el Tribunal de la CECA se inspira en cuanto a sus poderes y forma de funcionamiento en la justicia administrativa francesa.

${ }^{55}$ BOERGER-DE SMEDT, A., "La Cour de Justice ...”, p. 20.

${ }^{56}$ PENNERA, C., "The Beginnings of the Court of Justice...", p. 117.

${ }^{57}$ Artículo 77 TCECA: "La sede de las instituciones de la Comunidad será fijada de común acuerdo por los gobiernos de los Estados miembros".

${ }^{58}$ BITSCH, M.T., "La premiére institution...,", p. 130.
} 


\section{3.- PUESTA EN MARCHA DEL TRATADO Y PREVISIONES FINANCIERAS, COMERCIALES Y SOCIALES DEL TEXTO}

El tratado constitutivo de la Comunidad Europea del Carbón y del Acero entró en vigor el 23 de julio de 1952, momento a partir del cual se inició un período preparatorio que permitió adoptar las medidas necesarias para implementar el futuro mercado del carbón y del acero. Estas medidas que se encontraban en el convenio relativo a las disposiciones transitorias, uno de los anexos del tratado, se centraban fundamentalmente en la eliminación de obstáculos preliminares. Durante ese período, la Alta Autoridad tenía como misión informar y estudiar con los medios gubernamentales, profesionales y sindicales, los problemas que planteaba el establecimiento del mercado común, así como abolir los derechos de aduana, las restricciones cuantitativas a los cambios, las limitaciones a la entrega de divisas para la compra de productos, etc.

Era el título tercero del Tratado, que llevaba por título "Disposiciones económicas y sociales", el que se ocupaba de la política comercial en materia de carbón y acero, la financiación de la Comunidad y aspectos sociales relativos a la industria del carbón y del acero. Respecto a la política comercial común se reconocían a la Alta Autoridad competencias para ejecutar programas de inversiones o para fomentar la investigación sobre producción y consumo. Además, el texto recogía previsiones en el caso de reducción de la demanda o escasez de materiales- artículos 58 y 59 TCECA-. Para salvaguardar la libre competencia se prohibían acuerdos, concentraciones empresariales, subvenciones estatales y prácticas restrictivas que pudieran tener por efecto una competencia desleal o discriminatoria para alguno de los agentes del mercado ${ }^{59}$. En este sentido, se prohibía el establecimiento de condiciones discriminatorias respecto al transporte que restringieran o falsearan la libre competencia que propugnaba el texto. Como el mercado del carbón y del acero adoptaba la forma de unión aduanera, además de la la

\footnotetext{
${ }^{59}$ Por ejemplo, artículo 60 TCECA: “1. Quedarán prohibidas en materia de precios las prácticas contrarias a los artículos 2, 3 y 4, en particular: Las prácticas de competencia desleal, en especial las bajadas de precios meramente temporales o meramente locales tendentes, dentro del mercado común, a la adquisición de una posición de monopolio; [...]”
} 
armonización de la política comercial externa en materia de carbón y acero, se abolieron y prohibieron las barreras tarifarias internas, se establecieron condiciones comunes para comerciar con terceros países ajenos a la Comunidad y se estableció la fijación de precios bajo determinados supuestos ${ }^{60}$.

En el ámbito social la Alta Autoridad tenía entre sus funciones el fomento de la investigación en materia de seguridad en el trabajo de las industrias relacionadas con el carbón y acero ${ }^{61}$ - artículo 55 TCECA: "La Alta Autoridad deberá fomentar la investigación técnica y económica relacionada con la producción y el desarrollo del consumo del carbón y de acero, así como la seguridad en el trabajo de estas industrias. Organizará a este fin, los contactos adecuados entre los organismos de investigación existentes [...]"-. Además el tratado recogía medidas tendentes a mejorar las condiciones laborales de los trabajadores de las industrias afectadas por el tratado, así como se preveía la posibilidad de recurrir a la mano de obra de otros estados miembros de la Comunidad ${ }^{62}$-artículo 69: "1. Los Estados miembros se comprometen a suprimir toda restricción, por motivos de nacionalidad, respecto del empleo, en las industrias del carbón y del acero, de los trabajadores nacionales de uno de los Estados miembros de reconocida capacitación profesional en el campo del carbón y del acero, sin perjuicio de las limitaciones que resulten de las exigencias fundamentales de salud y de orden público. [...]"--

Las cuestiones sociales fueron una prioridad durante los primeros años de vida de la Comunidad, por ello se crearon ayudas a la readaptación del personal en paro de esas industrias ${ }^{63}$ - artículo 56 TCECA:

"Si la introducción, en el marco de los objetivos generales de la Alta Autoridad, de procedimientos técnicos o de instalaciones nuevas tuviere por efecto una reducción de excepcional importancia de las necesidades de mano de obra de la industrias del carbón y del acero, que acarreare en una o varias regiones dificultades particulares para el reempleo de la mano de obra que hubiere quedado disponible, la Alta Autoridad, a instancia de los

\footnotetext{
${ }^{60}$ MORATA, F., La Unión Europea. Procesos, actores y politicas. Barcelona, 1999, p. 24.

${ }^{61}$ BOSSUAT, G., "Les coopérations européennes pour la recherche scientifique et technique", Journal of European Integration History, 2006, vol. 12, number 2, pp. 5-10, p. 7.

${ }^{62}$ Título III. Disposiciones económicas y sociales.

${ }^{63}$ MECHI, L., "L'action de la Haute Autorité de la CECA dans la constuction de masisons ouvrières", Journal of European Integration History, 2000, vol. 6, number 1, pp. 63-88, p. 63.
} 
gobiernos interesados: [...] c) concederá una ayuda no reembolsable para contribuir: al pago de indemnizaciones que permitan a la mano de obra esperar hasta obtener una nueva ocupación, al pago de los trabajadores de indemnizaciones por gasto de traslado, a la financiación de la reconversión profesional de los trabajadores obligados a cambiar de empleo. [...]".

También se incluyeron ayudas a la investigación en el ámbito de la seguridad, la higiene, la medicina del trabajo, la financiación de programas sobre afecciones cardio-pulmonares o sobre contaminación atmosférica por las emisiones de la siderurgia, así como la construcción de viviendas para reemplazar campamentos de barracones provisionales o alojamientos insalubres ${ }^{64}$. Estas últimas, que no aparecían explícitamente previstas por el Tratado, sino que fueron objeto de una interpretación extensiva de los objetivos recogidos en el artículo 3 -entre los que se encontraba la mejora del nivel de vida de la mano de obra-, fueron de las medidas de carácter social que alcanzaron mayor éxito y que supusieron una significativa mejora de las condiciones de la mano de obra puesto que los problemas de vivienda eran urgentes, no solo porque en virtud de las estimaciones de la época, la guerra había destruido alrededor del 5 o el $6 \%$ de los edificios franceses e italianos, el $8 \%$ de las construcciones belgas y neerlandesas y el $20 \%$ de las alemanas, sino porque la mejora de las condiciones de habitabilidad de los trabajadores podía ayudar a la nueva organización a aumentar su popularidad en una situación política caracterizada por el fuerte poder de atracción sobre las masas que ejercían los movimientos comunistas. Otros argumentos, puramente económicos, animaron a Monnet y sus colaboradores a ocuparse de la cuestión, pues en el caso de trabajadores que residían lejos del lugar de trabajo los gastos de transporte, a menudo a cargo de las empresas, entrañaban un aumento sensible de los costes de producción ${ }^{65}$. Por todo lo anterior, se ejecutaron numerosas acciones para la protección del empleo, la mejora de las condiciones de higiene y seguridad en el trabajo, la readaptación de la mano de obra despedida y el desarrollo de la formación profesional. Así como se estudiaron salarios y horarios de trabajo en vigor en las industrias carboneras y siderúr-

\footnotetext{
${ }^{64}$ BITSCH, M.T., "La premiére institution...”, p. 136.

${ }^{65}$ MECHI, L., "L'action de la Haute Autorité ...", p. 64.
} 
gicas de los Estados miembros. En este sentido, el artículo 68 prohibía la bajada de salarios como medio de mejorar la competitividad:

"1. Sin perjuicio de las disposiciones siguientes, la aplicación del presente Tratado no afectará, por lo que respecta a las industrias del carbón y del acero, a las modalidades de fijación de los salarios y de las prestaciones sociales en vigor en los diferentes Estados miembros. [...]".

Respecto a las previsiones financieras del tratado, el texto reguló la puesta en marcha de tasas sobre la producción de carbón y acero para financiar las actividades de la Comunidad ${ }^{66}$. Estas, junto a los empréstitos en los mercados financieros fueron la fuente principal de financiación de la organización del carbón y el acero. La autonomía financiera fue una de las particularidades de la CECA y uno de los elementos esenciales de su independencia frente a los gobiernos. La Comunidad en lugar de obtener recursos, como la mayor parte de las organizaciones internacionales, a través de los fondos presupuestarios gubernamentales instituyó, en su texto constitutivo, lo que Lapié calificó como el primer impuesto europeo ${ }^{67}$. La tasa sobre el carbón y el acero que servía para financiar los gastos de funcionamiento de la Comunidad y para constituir un fondo de garantía quedó fijada en un porcentaje máximo de un $1 \%$ sobre la producción del carbón y acero, aunque no se llegó nunca a un nivel tan elevado. En un primer momento, dada la necesidad de constituir de forma rápida un fondo de garantía de cien millones de dólares, se impuso la tasa del 0,9\%. En mayo de 1955, cuando el fondo de garantía ya está prácticamente constituido, - los cien millones se alcanzaron en abril de 1956-, la República Federal de Alemania y Luxemburgo solicitaron una reducción pasando entonces a un $0,7 \%$ el 1 de julio de 1956 y a 0,45\% a partir del 1 de enero de 1957 . Todavía se reduciría más, a 0,35\% el 1 de julio de 1957 , después el $0,30 \%$, el 0,20\%, etc. ${ }^{68}$.

Tal y como se había previsto en la Declaración de 9 de mayo de 1950, el tratado CECA dejaba la puerta abierta a la adhesión de nuevos países al establecer el procedimiento de adhesión. La incorporación requeriría solicitud

\footnotetext{
${ }^{66}$ MORATA, F., La Unión Europea..., p. 24.

${ }^{67}$ LAPIÉ, Las tres Comunidades Europeas... p. 55.

${ }^{68}$ BITSCH, M.T., "La premiére institution ...”, p. 135.
} 
al Consejo, quien, tras pedir un dictamen a la Alta Autoridad, resolvería mediante un acuerdo unánime sobre la incorporación y sus condiciones ${ }^{69}$.

En diciembre de 1954 se firmó un acuerdo de asociación entre la Comunidad y el Reino Unido ${ }^{70}$ con una finalidad principalmente económica, al tratarse CECA de una organización de carácter comercial. Los británicos habían rechazado ser miembros pocos años antes, durante el gobierno laborista de Clement Attlee, al negarse a ceder el control de la industria del carbón y acero británica, bajo administración pública. Cuestión, esta última, que entraba en contradicción con el Tratado al impedir la existencia de subvenciones, monopolios o cualquier situación ventajosa respecto al resto de competidores. Sin embargo, a pesar del rechazo, otras voces, consideraban ventajosas algunas medidas que imponía el texto tales como la liberalización del mercado, la coordinación de las inversiones, la regulación de la producción en caso de bajada de precio o el intercambio de información entre actores de la industria. En definitiva, para algunos los problemas relativos a la industria del carbón, del hierro y del acero - básicos en la estructura industrial de Europa Occidental e interdependientes- podrían ser mejor resueltos por una autoridad internacional que de forma individual por cada Estado ${ }^{71}$.

Reino Unido acabó asociándose a la CECA lo que le suponía menores obligaciones comerciales que siendo miembro, pues el país insular gozaba de buenos accesos portuarios, de un carbón de buena calidad y de mayor productividad que el resto de países miembros. Las desventajas respecto a los seis países miembros eran que sus costes salariales eran mayores y que su no participación le podría dejar al margen de una comunidad o federación europea más amplia, a la que posteriormente Reino Unido pudiera querer adherirse. Se temía, por otra parte, que la ausencia británica incrementara la influencia de la República Federal de Alemania, no sólo en el ámbito económico, sino también político. Cuanto antes desplegaran los británicos su influencia sería más sencillo que la futura or-

\footnotetext{
${ }^{69}$ Artículo 98 TCECA.

${ }^{70}$ LAPIÉ, Las tres Comunidades Europeas... Pág. 60.

${ }^{71}$ LORD, C. "With but not of". Britain and the Schuman Plan, a reinterpretation", Journal of European Integration History, 1998, Vol. 4, Number 2., pp. 23-46, pp. 27-29.
} 
ganización coincidiera con sus preferencias. En este sentido, Reino Unido tenía necesidad de incrementar su acceso al mercado continental, pues exportaba menos de un $2 \%$ de su producción de carbón a los seis países miembros y únicamente cien mil toneladas de acero. Pero no siendo miembro evitaba otro riesgo: que los países firmantes de la CECA ofrecieran carbón y acero más barato a los países de la Commonwealth, lo que reduciría considerablemente las exportaciones británicas ${ }^{72}$.

\section{4.- BALANCE DE FUNCIONAMIENTO Y VIGENCIA}

El mercado común instaurado por la Comunidad Europea del Carbón y del Acero tuvo efectos económicos positivos para los países firmantes de forma rápida, tanto respecto a la bajada de precios como en la diversificación de productos o en la mejora de las condiciones de trabajo ${ }^{73}$. Sin embargo el logro más importante, como la reconciliación franco-alemana, mediante el establecimiento de una comunidad intereses entre ambos países, tuvo implicaciones mayores que las estrictamente económicas ${ }^{74}$. Además, la creación de la CECA permitió controlar la industria de Alemania Occidental, acercó al país germano a sus vecinos occidentales y preparó el camino para su rearme e integración en la estructura de defensa Occidental, la OTAN, en $1955^{75}$.

El éxito de una unión de países como la CECA que tuvo poderes limitados - fue una organización de carácter sectorial por lo que sus atribuciones quedaron circunscritas únicamente al sector del carbón y acero- pero cedidos por los Estados miembros en ejercicio de su soberanía parecía un buen método para conseguir la unión entre países en contraposición al Consejo de Europa o la OECE, en las que siendo su ámbito de aplicación mucho más amplio - al conjunto de la economía en el caso de la OECE - sus institucio-

\footnotetext{
${ }^{72}$ LORD, C. "With but not of"...", pp. 30-34.

${ }^{73}$ Véase por ejemplo HERNÁNDEZ ANDREU, J, Del 29 a la crisis asiática, Madrid, 1999, p. 86, o BOSSUAT, en las obras citadas en este trabajo.

${ }^{74}$ MORATA, F., La Unión Europea..., pp. 24 y 25.

${ }^{75}$ MUELLER, W., “The Soviet Union ...", p. 73.
} 
nes tenían unos poderes mucho más restringidos, dado que no se había producido una cesión de poderes soberanos a esas organizaciones -en ambos casos el poder decisorio lo ostentaban instituciones formadas por representantes gubernamentales de los Estados-. Sin embargo, según Bossuat, el fracaso en la ratificación de la Comunidad Europea de Defensa (CED) que fue un espejo del modelo CECA en el ámbito de la defensa demostró los límites de este método de integración ${ }^{76}$.

Argumentos usados para justificar que la CECA fue una organización fracasada se centran en que la organización quedó compuesta únicamente por seis países, sus ideólogos no habían contemplado la posibilidad de que Reino Unido no participara -aunque en poco tiempo se convirtió en país asociado a la Comunidad-, o por el hecho que hubieron de recortarse de forma considerable los poderes que se habían previsto para la Alta Autoridad y se añadieron otras instituciones -Consejo de Ministros, Asamblea o Tribunal de Justicia- que no habían sido inicialmente previs$\operatorname{tas}^{77}$. Gillingham hace un balance negativo de los primeros años de funcionamiento de la CECA:

"Everywhere output of the combustible continued to be regulated by quasi-official national cartels. Faced with the prospect of competition, the steel industry tightened informal alliances an even openly formed a new International Steel Cartel to regulate exports. Although the High Authority issued flurry of regulations governing price, marketing, investment, and so on, their only real function was to conceal its impotence in masses of verbiage, ${ }^{, 78}$.

La lucha contra los cárteles y concentraciones de empresas fue otro de los fracasos de la Comunidad, pues la Alta Autoridad no podía intervenir en empresas ya existentes en el momento de su creación, solo, en virtud del artículo 66 podía impedir nuevas concentraciones que dificultasen la competencia $^{79}$ :

\footnotetext{
${ }^{76}$ BOSSUAT, G., Histoire de l'Union européenne..., p. 165.

${ }^{77}$ BOSSUAT, G., Histoire de l'Union européenne..., p. 163.

${ }^{78}$ GILLINGHAM, J., “American Monnetism and ...", p. 29.

${ }^{79}$ BITSCH, M.T., "La premiére institution ...", p. 139.
} 
"1. Sin perjuicio de lo dispuesto en el apartado 3, requerirá la autorización previa de la Alta Autoridad toda operación que, en los territorios a que se refiere el párrafo primero del artículo 79 , y como resultado de la acción de una persona o de una empresa, de un grupo de personas o de empresas, tenga por sí misma por efecto directo o indirecto una concentración de empresas, una de las cuales al menos quede sujeta a la aplicación del artículo 80 , tanto si la operación se refiere a un mismo producto o a productos diferentes como si se efectúa mediante fusión, adquisición de acciones o del activo, préstamo contrato o cualquier otro medio de control. [...]".

La importancia de la organización acabó declinando con el paso del tiempo pues a finales de la década de los cincuenta tuvo que enfrentarse a una crisis carbonera de sobreproducción, ocasionada fundamentalmente por la aparición de nuevas fuentes de energía como el petróleo, gas o energía nuclear, que relegó el papel del carbón a un segundo lugar, así como a la importación de esta materia a terceros países no comunitarios, cuyos costes de producción eran menores, lo que hizo descender la competitividad del carbón europe ${ }^{80}$. Esta crisis afectó especialmente a Bélgica, quien instó a los países importadores de carbón, como Holanda o Italia, a limitar sus compras a países terceros en beneficio de los países de la Comunidad. Como consecuencia de esta situación, a principios de 1959 la Alta Autoridad propuso a los Estados miembros declarar un "estado de crisis manifiesta" para la industria del carbón. Según el artículo 58, esta declaración permitía instaurar un régimen cuotas de producción y reducir las importaciones provenientes de terceros países:

"1. En caso de contracción de la demanda, si la Alta Autoridad estimare que la Comunidad atraviesa un período de crisis manifiesta y que los medios de acción previstos en el artículo 57 no permiten hacer frente a la misma, deberá, previa consulta al Comité Consultivo y con el dictamen conforme del Consejo, establecer un régimen de cuotas de producción acompañado, en tanto fuere necesario, de las medidas previstas en el artículo $74 .[\ldots] "$

${ }^{80}$ ALTER, K. J, and STEINBERG, D., "The Theory and Reality of the European Coal and Steel Community", Buffet Center for International and Comparative Studies, Working Paper No. 07-001, January 2007, p. 7. 
Sin embargo, la decisión de declarar la situación de crisis debía obtener el dictamen conforme del Consejo de Ministros. Este último rechazó la medida al procederse a la votación: tres votos a favor y tres en contra ${ }^{81}$. Los tres países que votaron en contra fueron Francia, República Federal de Alemania e Italia, ya que ninguno tenía interés en poner en marcha un plan de acción contra la crisis $^{82}$.

La creación de nuevas Comunidades Europeas -CEEA, CEE-, a finales de los cincuenta, especialmente la Comunidad Económica Europea, con un ámbito de aplicación mucho más amplio, más la ya mencionada crisis carbonera y siderúrgica eclipsaron a la CECA que perduraría hasta el año 2002. En las disposiciones generales, título IV del Tratado, se establecía que la vigencia del texto sería de cincuenta años a partir de la entrada en vigor del mismo $^{83}$.

\footnotetext{
${ }^{81}$ BITSCH, M.T., "La premiére institution ...”, pp. 138-139.

${ }^{82}$ ALTER, K. J, and STEINBERG, D., "The Theory and...”, p. 7.

${ }^{83}$ Artículo 97 TCECA.
} 